\title{
UTILIZAÇÃO DOS CONCEITOS LEAN NA ETAPA DE CONDICIONAMENTO DE PLACAS NA ARCELORMITTAL TUBARÃO *
}

\author{
Mateus Costa Corona ${ }^{1}$ \\ Gladistone de Souza Guerra ${ }^{2}$ \\ Elcimar Silva Cunha ${ }^{3}$ \\ Luiz Alberto Andrade 4 \\ Vitor Bogaci Ney ${ }^{5}$ \\ Adriano Cesar Silva ${ }^{6}$ \\ Fabio Luiz Bassetti Cavalcante ${ }^{7}$
}

\begin{abstract}
Resumo
A ArcelorMittal Tubarão busca continuamente aumentar a sua produtividade, com isso, é necessário que as áreas de apoio a produção acompanhem este crescimento e sejam capazes de processar todo o volume produzido, sem comprometer a produção, a qualidade dos produtos e os prazos de entrega para os clientes. Os conceitos lean foram aplicados ao processo da Área de Condicionamento de Placas, com foco no aumento da produtividade e redução do lead time de processamento das placas.
\end{abstract}

Palavras-chave: Condicionamento de Placas; Metodologia Lean; ArcelorMittal Tubarão.

\section{LEAN CONCEPTS IN THE SLAB CONDITIONING STEP AT ARCELORMITTAL TUBARÃO}

\section{Abstract}

Arcelormittal Tubarão continually seeks to increase their productivity, therefore, it is necessary for the production support areas to keep up with this growth and be able to process all volume produced, without influencing on the product quality and lead time. The lean concepts were applied to the process of slabs conditioning area, focusing on increasing productivity and reducing the lead time of the slab processing.

Keywords: Slab finishing; Lean manufacturing; ArcelorMittal Tubarão.

1 Especialista de Controle Integrado de Qualidade de Produtos da ArcelorMittal Tubarão, Vitória, ES, Brasil.

2 Gerente da Área de Metalurgia da ArcelorMittal Tubarão, Vitória, ES, Brasil.

3 Gerente da Área de Acabamento de Placas e Bobinas a Quente da ArcelorMittal Tubarão, Vitória, ES, Brasil.

4 Supervisor de Operações da Área de Acabamento de Placas e Bobinas a Quente da ArcelorMittal Tubarão, Vitória, ES, Brasil.

5 Especialista de Controle Integrado de Qualidade de Produtos da ArcelorMittal Tubarão, Vitória, ES, Brasil.

6 Especialista de Engenharia Industrial da ArcelorMittal Tubarão, Vitória, ES, Brasil.

7 Supervisor de Operações da Área de Metalurgia da ArcelorMittal Tubarão, Vitória, ES, Brasil. 


\section{INTRODUÇÃO}

A ArcelorMittal Tubarão é uma usina integrada (figura 1) com capacidade produtiva de 7,5 milhões de toneladas de placas de aço por ano, 4,0 milhões para a produção interna de bobinas laminadas a quente e 3,5 milhões para clientes de todas as partes do mundo, sendo reconhecida mundialmente pela excelência em qualidade dos seus produtos, atendendo as mais diferentes aplicações do mercado, com destaque para os aços para estampagem (IF - Intersticial free), indústria automotiva, indústria naval, aços estruturais, aços para a indústria de petróleo e gás, etc.

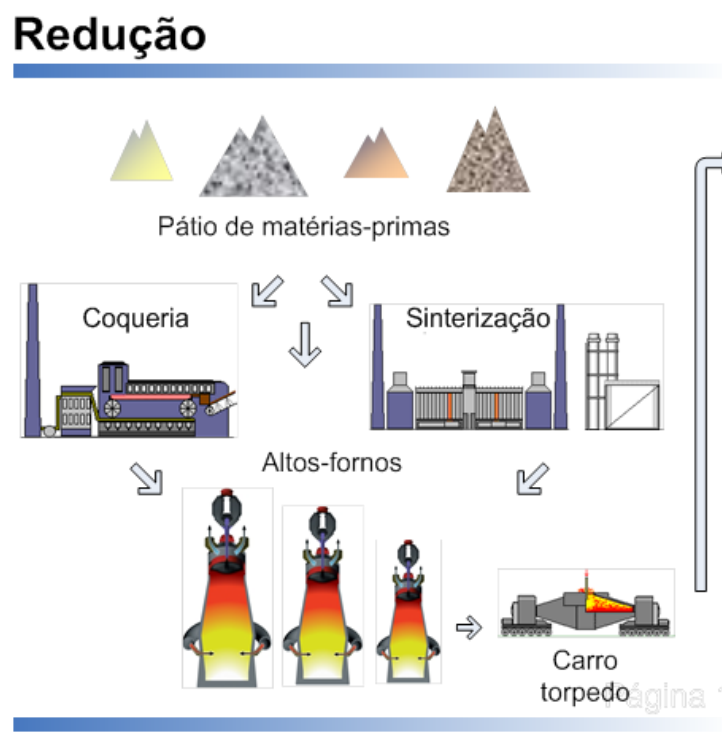

\section{Aciaria e Lingotamento}

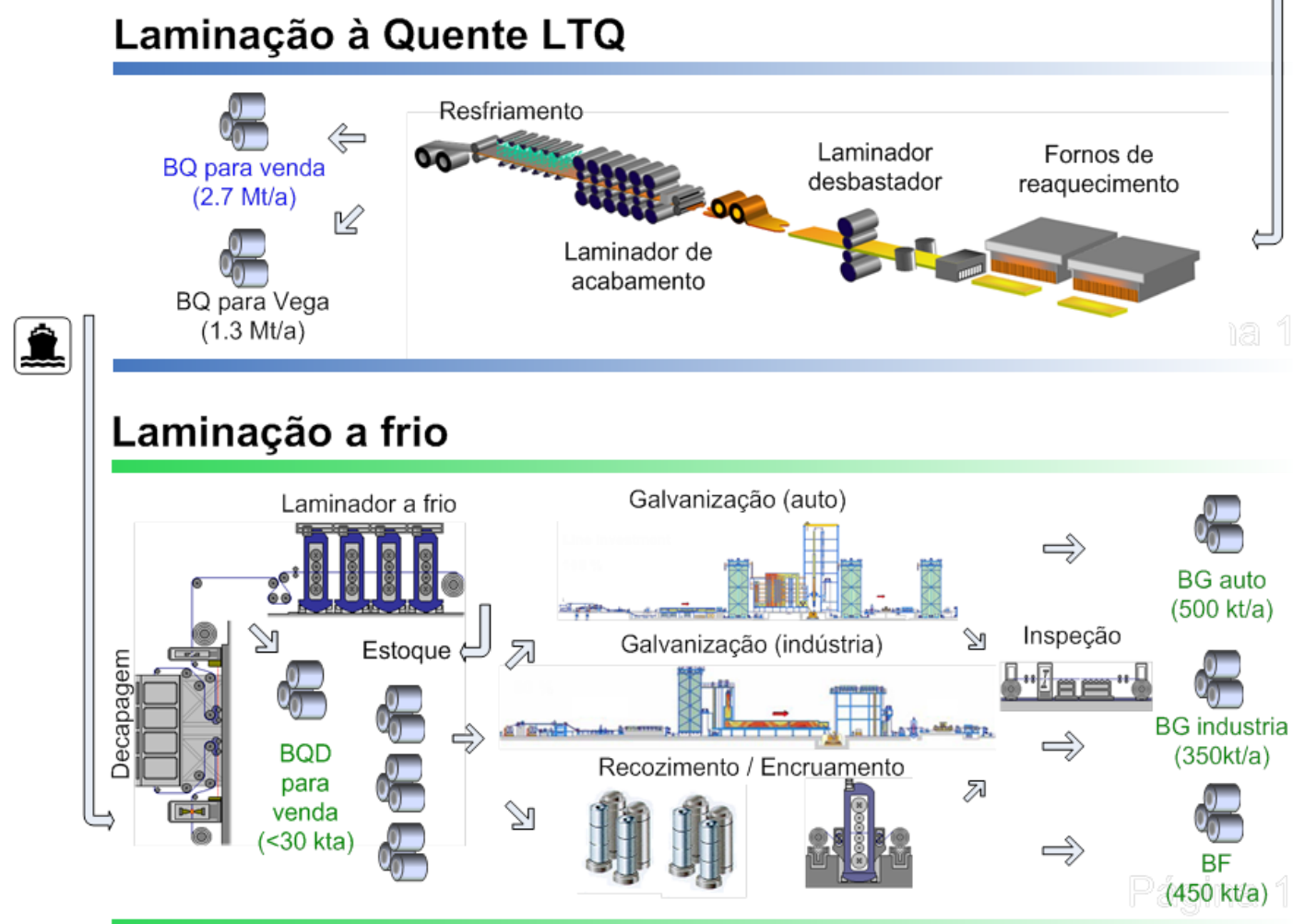

Figura 1. Fluxograma de produção da ArcelorMittal Tubarão.

Para garantir uma boa qualidade dos produtos (aplicação final) é importante que a placa esteja dentro das especificações exigidas pelos clientes, com composição 
química e dimensões dentro das tolerâncias, sem defeitos internos (trincas, segregações grosseiras, etc.), sem defeitos de forma (empenos, concavidade, convexidade, etc.), e com uma superfície bem acabada e livre de imperfeições. Para isso, é fundamental que as condições mecânicas da máquina de lingotamento contínuo estejam as melhores possíveis, assim como os parâmetros metalúrgicos e de processo. ${ }^{2,3}$

O Sistema de Gestão da Qualidade é fundamental para os bons resultados da empresa, que conta com certificações nas normas ISO 9001 e ISO/TS16949. Além da certificação que abrange toda a planta industrial, a ArcelorMittal Tubarão possui outras certificações de qualidade dos produtos para aplicação específicas (naval e estrutural). Atender aos requisitos dos clientes internos e externos, melhorar continuamente a eficácia do sistema de gestão, garantindo a qualidade de produtos e serviços, garantir a estabilidade dos processos e a redução de variabilidade, o estabelecimento, cumprimento e aprimoramento de padrões e promover constantemente ações que contribuam para a simplificação de atividades, visando o aumento da produtividade, são princípios básicos da política de gestão da qualidade da ArcelorMittal Tubarão.

O controle da qualidade dos produtos ArcelorMittal Tubarão (placa e bobina) é garantido pelos sistemas de monitoramento do processo de produção e pelas inspeções de qualidade realizadas ao longo das várias fases da cadeia produtiva. $A$ qualidade do produto final e a garantia para que o aço esteja em conformidade com as especificações dos clientes é obtido por meio da inspeção da produção. Apesar de todo monitoramento do processo e de suas variáveis, desvios de qualidade no produto são passíveis de ocorrer. Estes desvios, na maioria dos casos, podem ser corrigidos com operações de condicionamento. ${ }^{2,3,4}$

Entretanto, estas atividades aumentam os custos de produção e, na maioria das vezes, não agregam valor ao produto. Baseado nos fundamentos da melhoria contínua, na metodologia Lean e com foco no aumento do desempenho operacional, foi realizada uma avaliação do fluxo produtivo da área de condicionamento de placas, buscando identificar e atuar nas atividades e intervenções desnecessárias, que consomem recurso e tempo, sem agregar valor ao produto.

\subsection{Defeitos superficiais em placas produzidas pelo processo de lingotamento contínuo}

Os defeitos das placas semiacabadas, gerados no lingotamento contínuo, podem ser divididos em quatro tipos:

- Defeitos de forma (convexidade, abaulamento, estufamento, concavidade, etc.);

- Defeitos dimensionais (variação da largura, espessura e comprimento em relação ao especificado);

- Defeitos superficiais (figura 2) (corpo estranho, incrustação de carepa, inclusões de pó ou de refratário, colamento de aço no molde, duplo lingotamento, pele dupla, marca de equipamento ou marca de guia ou risco, marca de rolo, bolhas superficiais ou porosidade superficial, marcas de oscilação, trincas, etc.); 
- Defeitos internos (trincas, macro segregação, inclusões não metálicas, etc.). Os desvios de qualidade são identificados no momento da inspeção e, em alguns casos, podem ser corrigidos com operações de condicionamento, evitando que as placas com possíveis anormalidades possam chegar até os clientes. $1,2,3,4$

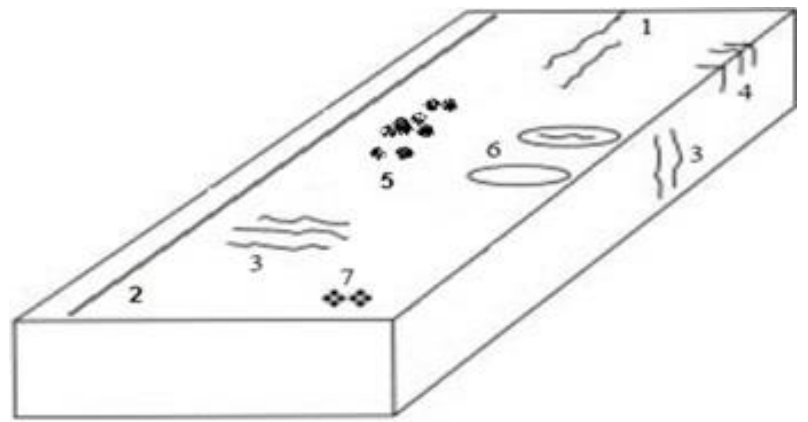

Figura 2. Defeitos superficiais: Trinca longitudinal (1); Marca de rolo / Marca de equipamento / Marca de guia / Risco (2); Trinca transversal (3); Trinca de quina (4); Incrustação de carepa; (5) Depressões (6); Inclusões de pó (7).

\subsection{Métodos de inspeção e condicionamento de placas de aço produzidas pelo processo de lingotamento contínuo}

A área de condicionamento é responsável por receber a produção do lingotamento contínuo e processar as placas de acordo com as suas instruções específicas, de resfriamento, inspeção, marcação, estocagem e despacho. $\mathrm{Na}$ inspeção, realizada após a etapa de resfriamento, é o momento onde são checados os parâmetros que conferem qualidade, no que diz respeito à dimensão, forma e superfície. Neste momento também são realizadas as instruções de condicionamento, conforme normas, padrões e especificação de fabricação de cada cliente. Os defeitos e imperfeições encontrados nas placas podem ser eliminados por escarfagem ou corte. ${ }^{1,2,3,4}$

\subsubsection{Procedimento de escarfagem de placas}

Alguns tipos de defeitos podem ser eliminados por procedimentos de escarfagem ou corte. Se for considerado que o defeito não pode ser eliminado, a placa semiacabada pode ser sucateada ou desviada para uma aplicação para o qual seja aceitável. A escarfagem das placas (figura 3) tem por finalidade eliminar defeitos superficiais e imperfeições que podem causar defeitos no produto final. ${ }^{2,3}$

O procedimento pode ser realizado parcialmente, atuando em defeitos pontuais, ou total, realizada em toda a superfície da placa. A exigência de aplicação do produto final é o principal fator levado em consideração na decisão de escarfar ou não uma placa. ${ }^{2,3}$

O procedimento pode ser realizado com três objetivos:

- Escarfagem investigativa, para verificar a existência de algum defeito superficial ou subsuperfícial na placa;

- Escarfagem preventiva, com objetivo de remover alguma região superficial da placa que possa ter sido afetada pela ocorrência de algum evento de qualidade (gerados automaticamente a partir de anormalidades ocorridas 
durante o processo de lingotamento contínuo, podem ser desclassificatórios ou condicionáveis em função da aplicação final);

- Escarfagem corretiva, com objetivo de retirar algum tipo de defeito superficial da placa, que possa prejudicar a qualidade do produto final. ${ }^{2,3}$

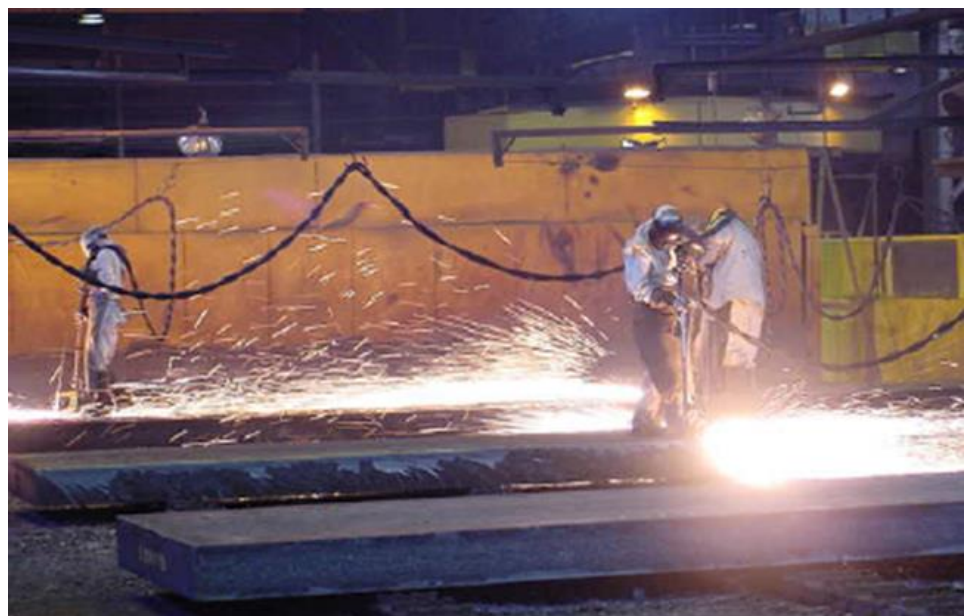

Figura 3. Procedimento de escarfagem de placas.

\subsection{Metodologia Lean}

A filosofia "Lean" (enxuto) tem como objetivo o aumento de produtividade e otimização do fluxo de processo, com foco em eliminar os desperdícios do processo. As atividades e processos que agregam valor ou não ao produto final podem ser divididas em três categorias:

- Atividades que agregam valor;

- Atividades que não agregam valor;

- Atividades que não agregam valor, porém são necessárias.

Já os desperdícios e atividades que não agregam valor, podem ser divididos em oito categorias (figura 4): Deslocamento, espera, sobre-processamento, produção excessiva, estoque, retrabalho, transporte e engajamento. ${ }^{5,6,7,8,9}$

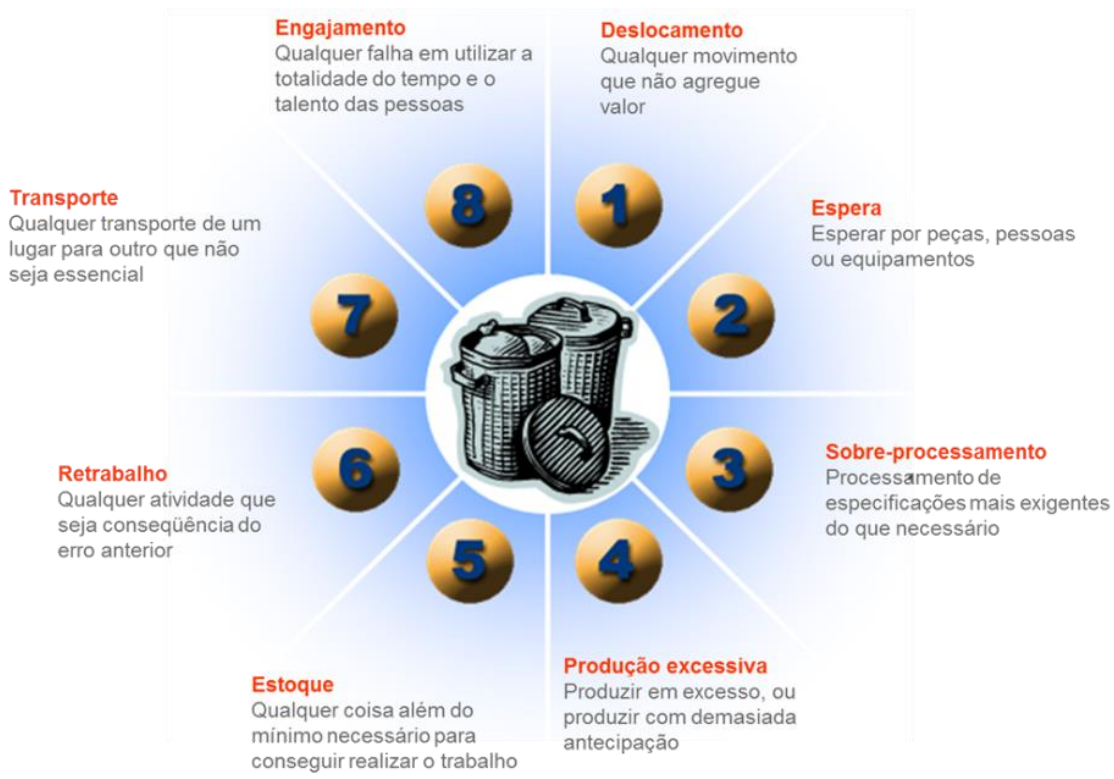

Figura 4. Formas de desperdício em processos. 


\section{MATERIAIS E MÉTODOS}

O estudo foi realizado em conjunto com os profissionais da área de condicionamento de placas, buscando aplicar ao máximo o conhecimento de cada um. Primeiramente foi realizado um mapeamento do estado atual da etapa de condicionamento e definição do escopo do projeto, em seguida, foram identificadas as possibilidades de melhorias e ajustes, e planejamento do estado futuro. Por fim, foram realizadas intervenções supervisionadas no processo que precederam o estabelecimento do estado futuro, com a implantação do novo fluxo de processo e revisão de padrões, contemplando as novas atividades e novos critérios. Mais detalhes nos tópicos a seguir.

\subsection{Identificação do estado atual e definição do escopo do projeto}

Foi realizado um mapeamento do fluxo de processo do condicionamento de placas, visando identificar as etapas necessárias e desnecessárias, as entradas e saídas, os retrabalhos e o lead time. Em seguida, buscou-se definir qual o objetivo de cada atividade, frequências, se estavam sendo desempenhadas de maneira correta, se agregavam valor ou não, se era necessárias ou não e qual era a sua influência na qualidade e no lead time de processamento das placas até a sua liberação para despacho para o porto. A capacidade de processamento da área, os tempos de execução das atividades e as pessoas envolvidas nos processos também foram avaliados. Foi especificado também o que cada cliente considera como valor para seu produto, ou seja, buscou-se identificar o que é importante para quem esta pagando pelo serviço/produto. Com base nas informações concebidas pelo mapeamento, foram identificadas várias oportunidades de melhorias e formas de desperdícios, tanto no processo quanto nas atividades. Iniciou-se então o planejamento do estado futuro, redefinindo o fluxo de processo da área e de algumas atividades, buscando tornar o processo mais enxuto e mais produtivo.

\subsection{Planejamento do estado futuro}

O estado futuro é o formato que se espera alcançar após a implantação das melhorias e das redefinições contempladas no processo e nas atividades, baseados na metodologia lean. Os mais diversos e possíveis cenários foram verificados por meio de simulações em planilhas de Excel. A maior preocupação neste momento foi verificar a influência das mudanças idealizadas, levando em consideração a capacidade e o tempo de processamento (lead time). Com a simulação também foi possível identificar os ganhos potenciais para o processo e os indicadores de desempenho. Após uma análise criteriosa, foi possível identificar atividades e tarefas dentro do processo que eram irrelevantes e que afetavam diretamente a produtividade e o custo da operação. Nesta etapa também foi realizada uma análise de riscos, contemplando a parte de segurança, saúde, qualidade e meio ambiente, ou seja, como estado futuro poderia influenciar nestas questões, com relação ao estado atual.

\subsection{Implementação do estado futuro e padronização atividades}

Após identificar o formato do estado futuro, foi feito um planejamento e um cronograma de implementação, com objetivos e metas a serem alcançados. Foi 
realizado um workshop, para alinhamento e treinamento, com as pessoas envolvidas nas atividades e no processo e apresentação do estado futuro. No período de implantação, os indicadores de desempenho eram avaliados após o final de cada turno, com objetivo de avaliar e validar a eficácia das novas metodologias e critérios adotados. Confirmado a eficácia do processo, os padrões operacionais de cada atividade foram então revisados. O monitoramento de eficácia, por meio dos Indicadores de desempenho da área, é realizado diariamente.

\section{RESULTADOS E DISCUSSÃO}

As ações de melhoria contínua realizadas na área de condicionamento de placas da ArcelorMittal Tubarão tiveram como principal objetivo aumentar a eficiência e produtividade da área, com foco na redução de:

- Sobre-processamento das placas (processamento de especificações mais exigentes do que o necessário);

- Retrabalho (qualquer atividade que seja consequência de um erro anterior);

- Excesso de movimentações (movimentos que não agregam valor e que são desnecessários).

\subsection{Redução do sobre-processamento e do retrabalho}

Pelo fato da ArcelorMittal Tubarão possuir um laminador de tiras a quente na unidade, com uma produção já consolidada, e uma filial em São Francisco do Sul (Vega), com processo de laminação a frio e galvanização, foi possível utilizar os parâmetros e critérios de qualidade das placas exigidos por estes processos como referência para o projeto, visando reduzir o sobre-processamento e o retrabalho. Estas referências foram levadas em consideração na etapa de especificação do que é considerado como valor para cada cliente e seus produtos. Após esta análise foi realizada uma revisão nos critérios de inspeção dos defeitos superficiais, levando sempre em consideração a aplicação do produto final e o não comprometimento de sua qualidade.

Observou-se durante o mapeamento do estado atual que estava havendo um tratamento excessivo no que se diz respeito à qualidade superficial das placas, ou seja, foram identificadas várias formas de sobre-processamento. Estas intervenções eram muito além do que era necessário, acrescentando custo e aumentando o tempo de processamento (lead time).

Foi observado também que a rota principal do fluxo de processo não favorecia a produção, devido principalmente a existência de diversos pontos de execução da mesma atividade e a possibilidade de uma mesma placa passar várias vezes por várias etapas do fluxo (fluxo não contínuo), gerando retrabalho várias vezes. Verificado estes fatos, foi planejado para o estado futuro um fluxo de produção com uma rota principal contínua, desviando as placas com necessidade de intervenção para áreas específicas e sem necessidade de retornarem ao fluxo principal.

A área de condicionamento da ArcelorMittal Tubarão processa, em média, $57 \%$ das placas produzidas mensalmente, o restante da produção (43\%) segue via rota direta para o laminador de tiras a quente (LTQ). O gráfico (figura 5) mostra, com relação ao 
ano de 2015, uma redução de aproximadamente $50 \%$ no percentual de placas escarfadas no ano de 2016 e aproximadamente 75\% em 2017.

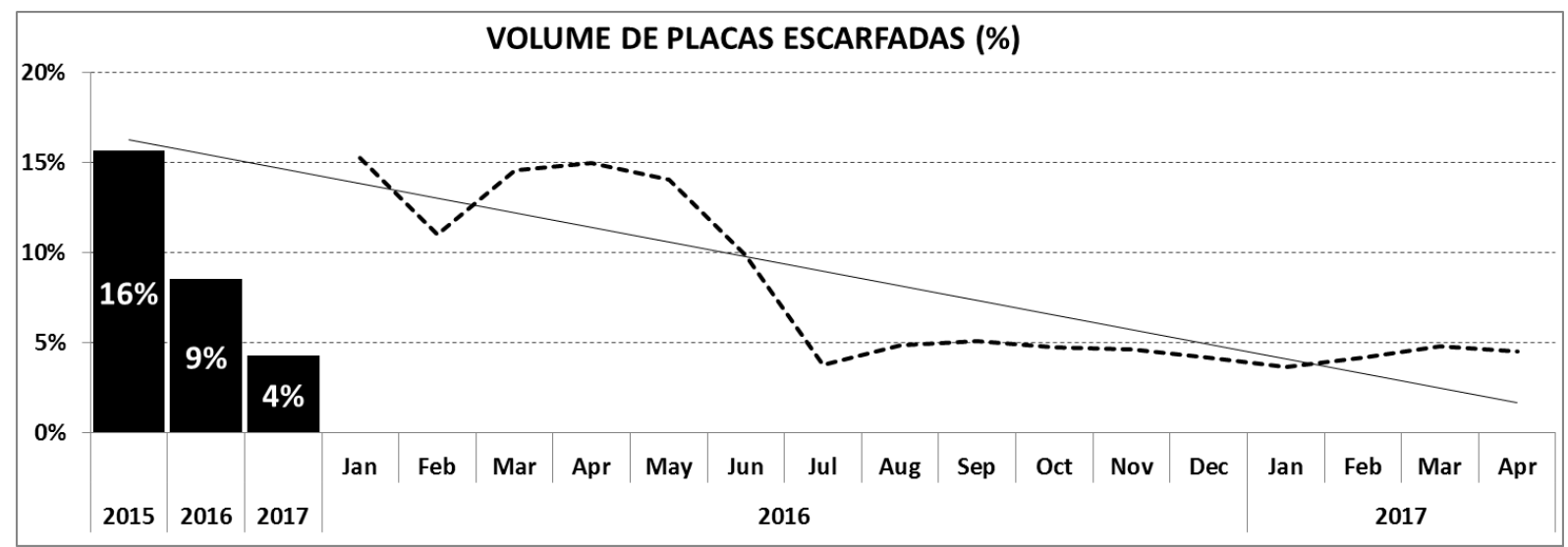

Figura 5. Indicadores de desempenho - Volume de placas escarfadas.

Este resultado foi alcançado devido a um conjunto de ações realizadas pelas seguintes áreas:

- Unidade Técnica da Aciaria e de Operação e Manutenção do Lingotamento Contínuo, que estão sempre buscando manter uma estabilidade das condições ideias de processo e produção;

- Área de Metalurgia e Controle da Qualidade de Produto, que mantém uma gestão dos indicadores de desempenho e avalia constantemente as condições de produção e qualidade dos produtos de acordo com os critérios de cada cliente;

- Área de Condicionamento de Placas, responsável por realizar todas as atividades de inspeção, condicionamento das placas e gestão dos indicadores de desempenho.

- Área de Engenharia Industrial, responsável pelos estudos de avaliação de capacidade da área, tempos e métodos de execução de tarefas e avaliação do cenário do estado futuro.

\subsection{Redução do Lead time de processamento das placas}

Algumas ações foram tomadas com o objetivo de reduzir o tempo de permanência das placas na etapa de condicionamento (tempo entre o final da produção da placa no lingotamento contínuo e a sua liberação de despacho para o porto). A seguir estão as principais ações que contribuíram para o bom resultado obtido:

- Adequação dos métodos de resfriamento (principal fator identificado que afetava o lead time), tornando possível o resfriamento das placas em um menor tempo;

- Utilização da ferramenta de gestão andon, com torres de luzes que indicam para os inspetores de qualidade, para os operadores de corte e para os operadores de ponte rolante, se a placa já teve seu processamento concluído ou que o berço de corte está disponível para receber outra placa;

- Aumento da efetividade das instruções de corte, evitando que a placa seja movimentada para área de corte sem que seja necessário;

- Ampliação da cultura de "multifunções", onde um operador seja capaz de realizar mais de um tipo de função; 
- Realização de kaizen e 5S, onde os operadores contribuíram com ideias para melhorias no processo de corte a gás das placas e organização da área, o que permitiu um aumento na produtividade, com maior capacidade de corte em menos tempo.

Com relação ao ano de 2015, em 2016 e 2017 foi observada uma redução de aproximadamente $30 \%$ no lead time de processamento das placas, na etapa de condicionamento.

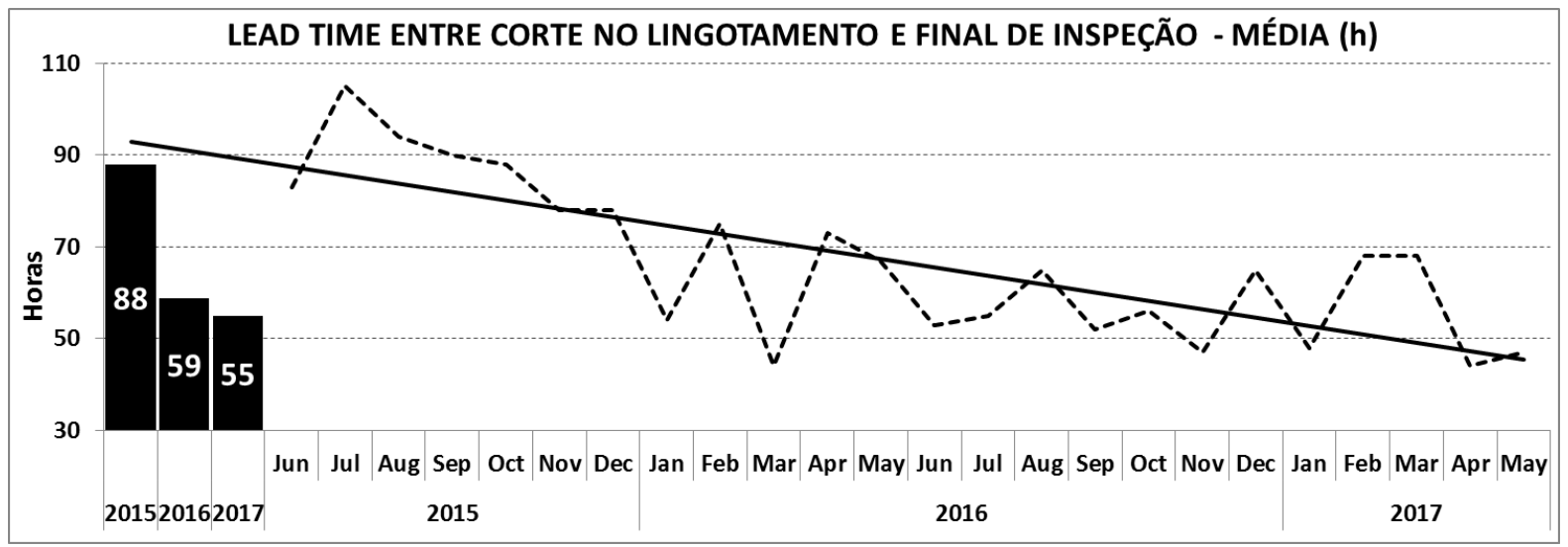

Figura 6. Indicadores de desempenho - Lead time entre o final de corte da placa no lingotamento contínuo e o final de inspeção na área de condicionamento.

\section{CONCLUSÃO}

O novo modelo de operação e as novas condições tornaram a área mais produtiva e mais eficiente, com menos desperdícios e com disponibilidade e agilidade para aproveitar oportunidades de preenchimento da carteira de pedidos. Outros ganhos como a redução do lead time de pedidos, redução de custos, redução do índice de retrabalho e redução de perdas metálicas também foram identificados.

\section{REFERÊNCIAS}

1 Carvalho, José L. R. Lingotamento Contínuo de placas de aço. São Paulo: Associação Brasileira de Metalurgia e Materiais; 1988.

2 Rizzo, Ernandes M. da Silveira. Introdução aos processos de lingotamento dos aços. São Paulo:, Associação Brasileira de Metalurgia e Materiais; 2006.

3 Perim, Carlos Alberto. Curso de Lingotamento Contínuo de Placas. São Paulo: Associação Brasileira de Metalurgia e Materiais, 2010.

4 Silva, Guilherme F. B. Lenz e. Lingotamento Contínuo e Defeitos de Laminação. São Paulo: Departamento de Engenharia Metalúrgica e de Materiais - Escola Politécnica / Universidade de São Paulo; 2010.

5 NAZARENO, R. R. Desenvolvimento e aplicação de um método para implementação de sistemas de produção enxuta. Dissertação de Mestrado, USP, São Carlos, 2003.

6 Junior, Alvair Torres; Costa, Tânia. Inovação como estratégia - o desenvolvimento lean de produtos e processos. 2017 [acesso em 15/05/2017]. Disponível em: http://www.lean.org.br/artigos/504/inovacao-como-estrategia-\%E2\%80\%93-odesenvolvimento-lean-de-produtos-e-processos.aspx 
7 Busato, Lírio; Ferro, José Roberto. Usando o lean coaching em busca da gestão lean. 2016 [acesso em 15/05/2017]. Disponível em: http://www.lean.org.br/artigos/461/usando-o-lean-coaching-em-busca-da-gestaolean.aspx

8 Lovro, Arthur. Aplicação do pensamento Lean no Desenvolvimento de Produtos. 2007 [acesso em 15/05/2017]. Disponível em: http://www.lean.org.br/artigos/67/aplicacao-dopensamento-lean-no-desenvolvimento-de-produtos.aspx

9 Nishida, Lando. Reduzindo o lead time no desenvolvimento de produtos através da padronização. 2007 [acesso em 15/05/2017]. Disponível em: http://www.lean.org.br/artigos/74/reduzindo-o-lead-time-no-desenvolvimento-deprodutos-atraves-da-padronizacao.aspx 